\title{
Planning and Analyzing Transit Networks: An Integrated Approach Regarding Requirements of Passengers and Operators
}

Markus Friedrich, Transport Planning Systems Unit, PTV

Thomas Haupt, Transportation Modeling Unit, PTV Klaus Noekel, Research and Development Unit, PTV

\section{$\overline{\text { Abstract }}$}

Providing an equally sufficient and efficient transit service requires careful planning and permanent monitoring of service quality, operating costs, and revenues. These requirements need a model that is capable of determining impacts on passengers as well as operators. Additionally, it is important to provide suitable and powerful methods to design and to modify the transit network. The transport planning software VISUM attempts to fulfill these requirements. In contrast to conventional Geographic Information Systems (GIS), which are extended to provide specific functionality for transit planning, VISUM is a comprehensive transportation model with additional GIS functionality. It seeks to fill the gap between conventional GIS programs and vehicle scheduling programs. 


\section{Introduction}

Demands for a competitive public transport that offers alternatives to private transport with minimal public subsidies call for a planning process that considers the impacts on passengers and operators. To passengers, good service quality means:

- short travel time,

- minimum number of transfers,

- good service frequency, and

- reasonable fares.

Operators and transit agencies must provide service in an economically efficient way. They need to monitor the performance of the existing service and forecast the impact of proposed measures. The operator, for example, needs to know the:

- required fleet size,

- operating costs,

- revenues from tickets, and

- cost coverage that indicates whether public subsidies are necessary.

These requirements of passengers and operators describe the fundamental conflict in transit planning. To solve this conflict, the transport planner needs to find an acceptable balance between two incompatible planning objectives: the maximization of service quality and the minimization of operational costs and public subsidies. For this complex planning task, transport planners started to apply software approximately three decades ago. Today, they can select from a variety of software tools for strategic planning, the most common of which are Comprehensive Transportation Models (CTMs) and GIS.

\section{CTMs versus GIS}

CTMs (e.g., Emme/2, Trips, TRANPLAN) were developed specifically for transport planning purposes. They connect travel demand data and supply data to determine traffic flows through the network. At the core of the models are an assignment procedure distributing travel demand onto the link network. In the beginning, most models focused only on private transport. Extensions to cover public transport often adapted modeling techniques for private transport. 
As a result, the models often tended to lack appropriate assignment procedures for public transport (i.e., timetable-based assignment procedures) and hardly considered aspects of transit operation.

GIS (e.g., MapInfo, ARC/INFO) is widely used for a broad range of purposes. Providing a user-friendly environment, it is applied to manage, analyze, and display geographical information by connecting database tables with geographical objects. Examples of GIS applications in the field of transportation include building and maintaining road databases and determining the accessibility of transit stops. Since standard GIS functionality does not cover specific transport aspects, GIS users all over the world are more or less successfully trying to adapt GIS according to their planning requirements. For instance, the TransCAD system is often used for transport planning purposes. TransCAD offers an alternative to CTM by combining GIS and transportation modeling capabilities.

\section{The VISUM Approach}

This article examines the transit features supported by the transport planning software VISUM (Friedrich 1998, 1999), which is part of the PTV VISION transportation software suite (PTV 1999). VISUM is a software program for planning and analyzing transportation networks (Figure 1). It provides specific functionality for public transport to help analyze and evaluate an existing or a proposed public transport service from the perspective of operators as well as passengers. By providing additional GIS functionality, VISUM fills the gap between conventional GIS, CTM programs, and vehicle and crew scheduling systems.

The development of VISUM continues to be strongly influenced by the needs of European transit authorities and operators. With the introduction of competition and the privatization of state-owned railway companies (Meyer 1997), many European transport companies were reorganized. Simultaneously, an increasing emphasis was placed on improving the attractiveness and efficiency of the service. Consequently, transportation planning software was expected to meet the following requirements:

- Multimodal transport model: Since the main challenger of transit operators is not the competing operator but private car transport, transport 


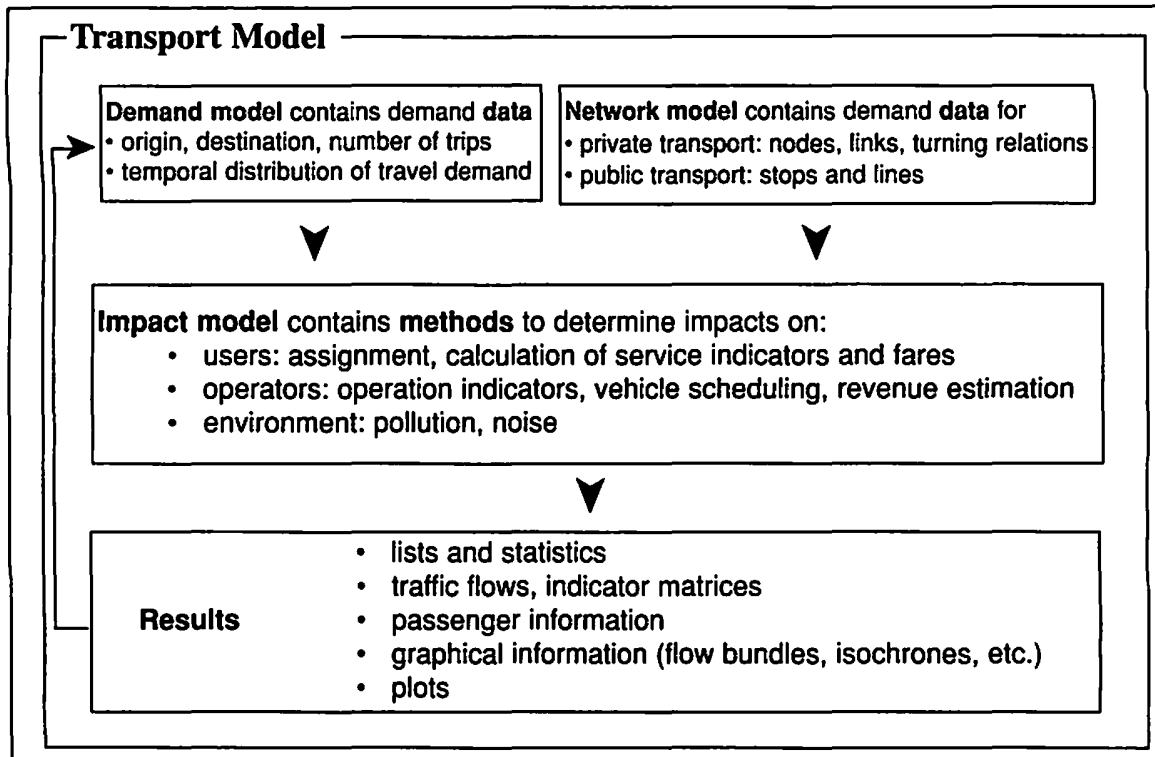

Figure 1. VISUM-comprehensive transport model and its submodels

models must integrate private and public supply systems in order to explore all the potentials for passengers.

- Continuous control: Transportation planning software is expected not only to assist during the planning process, but also to constantly monitor the performance of a transit system. Results of line-costing calculations serve as a continuous input for planning.

- Modeling of fare systems: Transparent and competitive fares are an essential prerequisite for success. This requires a model that allows the user to define fare zones and different types of tickets in order to estimate and optimize revenues. Transit networks with an integrated fare system depend on methods for distributing revenues onto lines of individual operators.

- Modeling of large networks: Railway companies and national transport planning agencies are in the process of establishing network models that incorporate precise line and timetable information on a nationwide or continent-wide level. 
VISUM attempts to cover these requirements. The specific transit features supported by VISUM include:

- Network model: VISUM offers a network model compatible with GIS as well as passenger information systems or vehicle and crew scheduling systems. As a result, it can combine geographical link network data and timetable data in an integrated network model.

- Fare model: VISUM provides a fare model to estimate revenues from ticket fares. The model supports distance-based as well as zone-based fares.

- Design process: VISUM provides functionality supporting the design process and assisting the planner in finding new solutions (e.g., by "drawing" the line-route on the screen).

- Service quality: VISUM includes specific assignment procedures for public transport that apply search algorithms similar to passenger information systems. This allows the user to examine the impacts on passengers by calculating essential service indicators and travel costs for each origin and destination (O-D) pair (journey time, waiting time, number of transfers, service frequency, fare).

- Line costing: VISUM supports line-costing calculations that state the profit or loss on individual transit lines regarding costs and revenues.

- Routes: VISUM offers the unique ability to store the routes of all passenger trips during assignment. Routes are most useful for postassignment analysis of traffic flows and the calculation of fares.

- Areas: VISUM can aggregate performance indicators as well as cost and revenue indicators for user-defined areas (e.g., traffic zones).

VISUM includes a demand model, VISEM, that estimates and forecasts mode-specific O-D matrices. The two basic features of VISEM are the classification of the population into behaviorally homogeneous population groups and the generation of trip chains derived from activity chains. For a more detailed description see Fellendorf et al. (1997). 


\section{Network Model}

The network model describes the supply side of the transport system consisting of several supply systems (Figure 2). Each supply system belongs to either the private transport (PrT) or public transport (PuT) mode, and uses one specific means of transport (car, truck, bike, bus, train, etc.). The combination of mode and means defines the system's characteristics determining a set of rules for the operation of the vehicles. The actual speed of individual transport vehicles is influenced by the network's capacity whereas transit vehicles operate according to their timetables. The requirements of an integrated network model for private and public transport influence the design of the network objects.

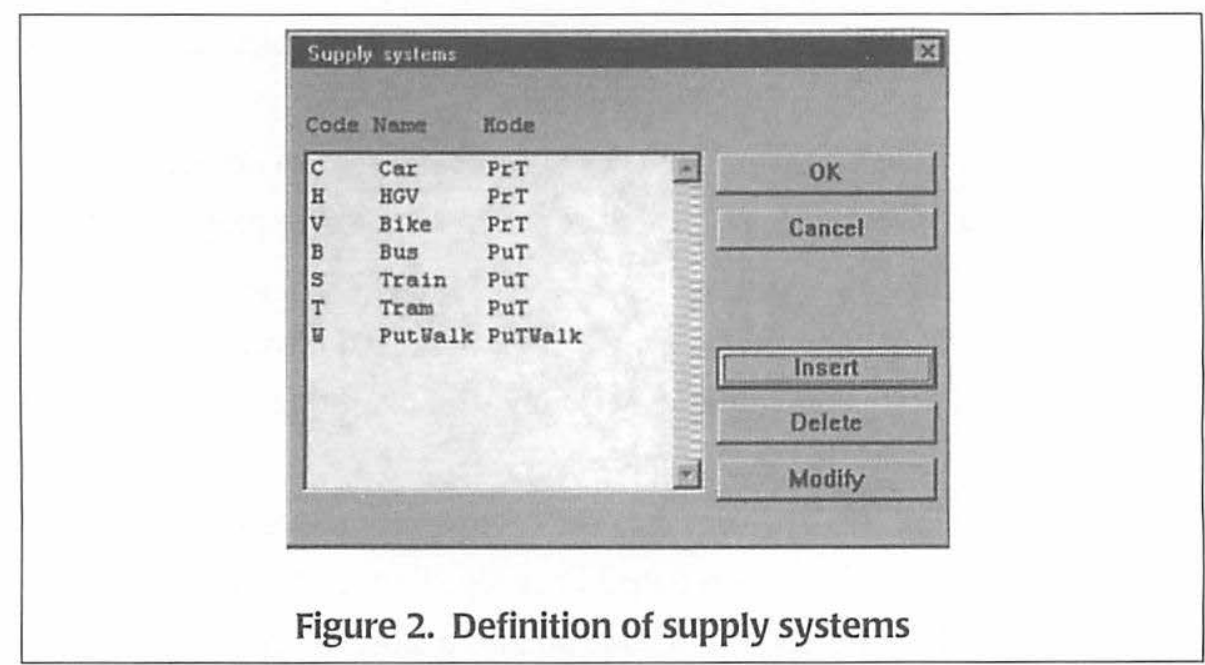

Source: Screen shot from VISUM 6.

- Nodes can represent intersections and/or public transport stops.

- Link attributes describe speed and capacity for private transport and carry default values for the running time of public transport vehicles.

- Turning relations penalize turning movements for private transport during assignment and define junctions for the construction of transit lines.

- Transit lines may use only links that are suitable for vehicles of the particular system. 


\section{Transit Lines}

A transit line has a particular line name and usually serves two directions. It may include one or several line variants (sublines) that show different lineroutes or running times between stops. A set of vehicle trips (service) defines the timetable, which can be calculated from the departure time of any vehicle trip at the origin stop and the running times between stops. A line belongs to one supply system and can, therefore, use only links that are permitted for this supply system (e.g., a bus may use only certain links of the road network while a train may use only the rail network). Each vehicle trip uses a defined type of vehicle that can carry information on vehicle-specific costs. Figure 3 shows a simple network with one bus line (Bus1). Figure 4 lists all relevant tables that are necessary to describe this network in a relational database.

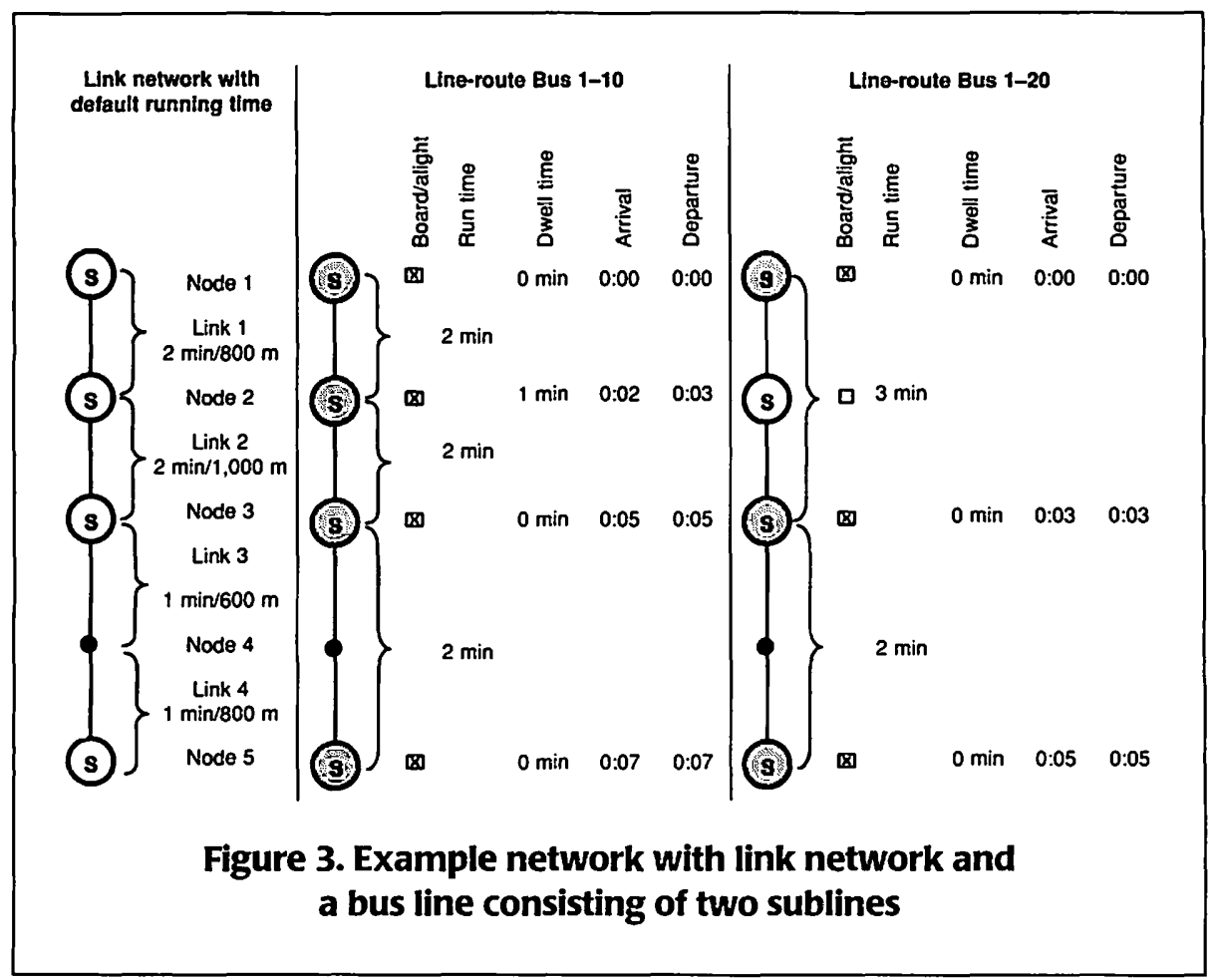




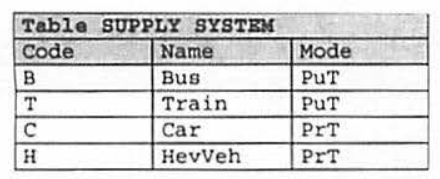

\begin{tabular}{|c|c|c|c|c|}
\hline NodeNo & Name & $\mathrm{x}$-Coord & $Y$-Coord & Stop \\
\hline 1 & Node1 & $\ldots$ & & 図 \\
\hline 2 & Node2 & & & 图 \\
\hline 3 & Node 3 & & & 图 \\
\hline 4 & Node4 & & & $\square$ \\
\hline 5 & Node 5 & & & 図 \\
\hline
\end{tabular}

\begin{tabular}{|c|c|c|c|c|c|c|}
\hline 21 & & & & & & \\
\hline LinkNo & FromNode & ToNode & Length & Supplysystem & PrT-Capacity & RunTime (Bus) \\
\hline 1 & 1 & 2 & 800 & $B, C, H$ & $\ldots$ & $120 \mathrm{~s}$ \\
\hline 2 & 2 & 3 & 1000 & $\mathrm{~B}, \mathrm{C}, \mathrm{H}$ & & $120 \mathrm{~s}$ \\
\hline 3 & 3 & 4 & 600 & $B, C, H$ & & $60 \mathrm{~s}$ \\
\hline 4 & 4 & 5 & 8000 & $B, C, H$ & & $60 \mathrm{~s}$ \\
\hline
\end{tabular}

\begin{tabular}{|l|l|r|r|r|r|r|}
\hline Table VRHICLB \\
\hline VehTypeNr & Name & TotalCapacity & SeatCapacity & Cost/Hour & Cost/Km & Cost/Veh \\
\hline 1 & Midibus & 40 & 20 & 42,00 & 1,00 & 100 \\
\hline 2 & Standardbus & 90 & 35 & 42,00 & 1,50 & 150 \\
\hline
\end{tabular}

\begin{tabular}{|c|c|c|c|}
\hline Name & Variant & Direction & Supplysystem \\
\hline BUS1 & 1 & I (inbound) & B \\
\hline BUS1 & 1 & 0 (outbound) & B \\
\hline BUS1 & 2 & I (inbound) & $B$ \\
\hline BUS1 & 2 & o (outbound) & B \\
\hline
\end{tabular}

\begin{tabular}{|c|c|}
\hline \multicolumn{2}{|l|}{ Table OPBRATOR } \\
\hline OperatorNI & Name \\
\hline 1 & Urban Operator \\
\hline 2 & Railway Company \\
\hline
\end{tabular}

\begin{tabular}{|c|c|c|c|c|c|c|c|c|}
\hline Name & Variant & Direction & NodeNr & Board & Aliaht & Arrival & Departure & Length \\
\hline BUS1 & 1 & 0 & 1 & 㘣 & 图 & $00: 00: 00$ & $00: 00: 00$ & 0 \\
\hline BUS1 & 1 & 0 & 2 & 国 & 图 & $00: 02: 00$ & $00: 03: 00$ & 800 \\
\hline BUS1 & 1 & 0 & 3 & 図 & 因 & $00: 05: 00$ & $00: 05: 00$ & 1000 \\
\hline BUS1 & 1 & 0 & 4 & 口 & $\square$ & $00: 00: 00$ & $00: 00: 00$ & 600 \\
\hline BUS1 & 1 & 0 & 5 & 图 & 図 & $00: 07: 00$ & $00: 07: 00$ & 800 \\
\hline BUS1 & 2 & 0 & 1 & 图 & 图 & $00: 00: 00$ & $00: 00: 00$ & 0 \\
\hline BUS1 & 2 & 0 & 2 & $\bar{\square}$ & $\square$ & $00: 00: 00$ & $00: 00: 00$ & 800 \\
\hline BUS1 & 2 & 0 & 3 & 国 & 図 & $00: 03: 00$ & $00: 03: 00$ & 1000 \\
\hline BUS1 & 2 & 은 & 4 & $\square$ & 므 & $00: 00: 00$ & $00: 00: 00$ & 600 \\
\hline BUS1 & 2 & 0 & 5 & 凶 & 図 & $00: 05: 00$ & $00: 05: 00$ & 800 \\
\hline
\end{tabular}

$\mid$\begin{tabular}{l|c|c|c|c|c|}
\hline Table TIMETABL \\
\hline Name & Variant & Direction & Departure & VehTypeNr & OperatorNr \\
\hline BUS1 & 1 & 0 & $6: 00: 00$ & 2 & 1 \\
\hline BUS1 & 1 & 0 & $6: 20: 00$ & 2 & 1 \\
\hline BUS1 & 1 & 0 & $6: 40: 00$ & 2 & 1 \\
\hline BUS1 & 2 & 0 & $6: 10: 00$ & 2 & 1 \\
\hline BUS1 & 2 & 0 & $6: 30: 00$ & 2 & 1 \\
\hline BUS1 & 2 & 0 & $6: 50: 00$ & 2 & 1 \\
\hline
\end{tabular}

Figure 4. Description of example network in a relational database 
- Table SUPPLY SYSTEM defines name and mode of each supply system.

- Table NODE contains the attributes of nodes that represent intersections or stops.

- Table LINK describes the link network. Each link is defined by two nodes and several input attributes (e.g., link length, permitted supply systems, free-flow speed, and capacity for private transport). The default running time for public transport systems is used during the interactive construction of a transit line in order to create a default timetable.

- Table VEHICLE defines types of transit vehicles. The capacity attributes allow the calculation of a line's saturation; the cost attributes are necessary to determine operating costs.

- Table SUBLINE defines lines and line variants.

- Table OPERATOR lists transit operators.

- Table LINE-ROUTE specifies a sequence of nodes and stops with running time between stops for each subline.

- Table TIMETABLE lists vehicle trips described by a departure time at the origin stop, a vehicle type, and an operator.

\section{Creating a Network Model}

Creating network models used to be a time-consuming task. The ability to obtain or purchase digital data opened new ways to build comprehensive and accurate network models. Main sources for multimodal network models are digital link network data (e.g., NavTech), which need to be connected with transit data containing information on transit stops, line-routes, and timetables. This involves a three-step process:

1. Import link network data using an interface to a GIS or relational database.

2. Import transit stops and match transit stops with nodes of the link network. The matching process geocodes the transit stops. Where transit stops do not find corresponding nodes, it is necessary to split links.

3. Import line-routes and timetables from the transit source. The transit source can be passenger information systems (e.g., Hafas, EFA), transit databases (e.g., Transmodel, UITP 1996), or vehicle scheduling systems 
(e.g., HASTUS, MICROBUS, INTERPLAN). Since these sources generally store only served stops with the line-route, the import process automatically inserts nodes into the line-route, to represent either intersections or through stops.

For importing and exporting network data, VISUM provides integrated interfaces to ASCII files (comma-separated values), relational databases (Microsoft Access, Oracle), and spreadsheets. Transit data can be accessed by product-specific interfaces or standardized transit databases.

\section{Interactive Construction of Transit Lines}

The main task of the planning process is the development of new solutions. Although new solutions may be generated through optimization algorithms, most solutions are still developed using the planner's creativity and experience, because the complex interdependencies within a transport system cannot be described appropriately through an objective function. Many practitioners spend a great amount of time modifying network data. On an extreme level, one can argue that as long as a planner prefers pen and paper for developing a first draft of a public transport network, the user interface of the modeling software needs to be improved.

In designing a transit line, a planner ideally wants to "draw" the lineroute on the screen. Operation aspects favor a line length that produces effective round-trip times and a stop sequence that ensures a sufficient catchment area. Passengers want fast, direct, and frequent line service with timed transfers.

The VISUM network editor provides a method to meet these requirements. In order to define a line-route, the user simply marks the two terminals of a transit line by a mouse click. Based on the link infrastructure, VISUM proposes a complete line-route with running times and distances. The proposed line-route may be subsequently modified by merely dragging parts of the line onto other links (Figure 5). Using a standardized timetable (e.g., peak hours/off-peak hours, 10-minute/20-minute headways) and an O-D matrix, it is possible to continuously inform the planner about the line's performance by displaying essential indicators in a status window: 


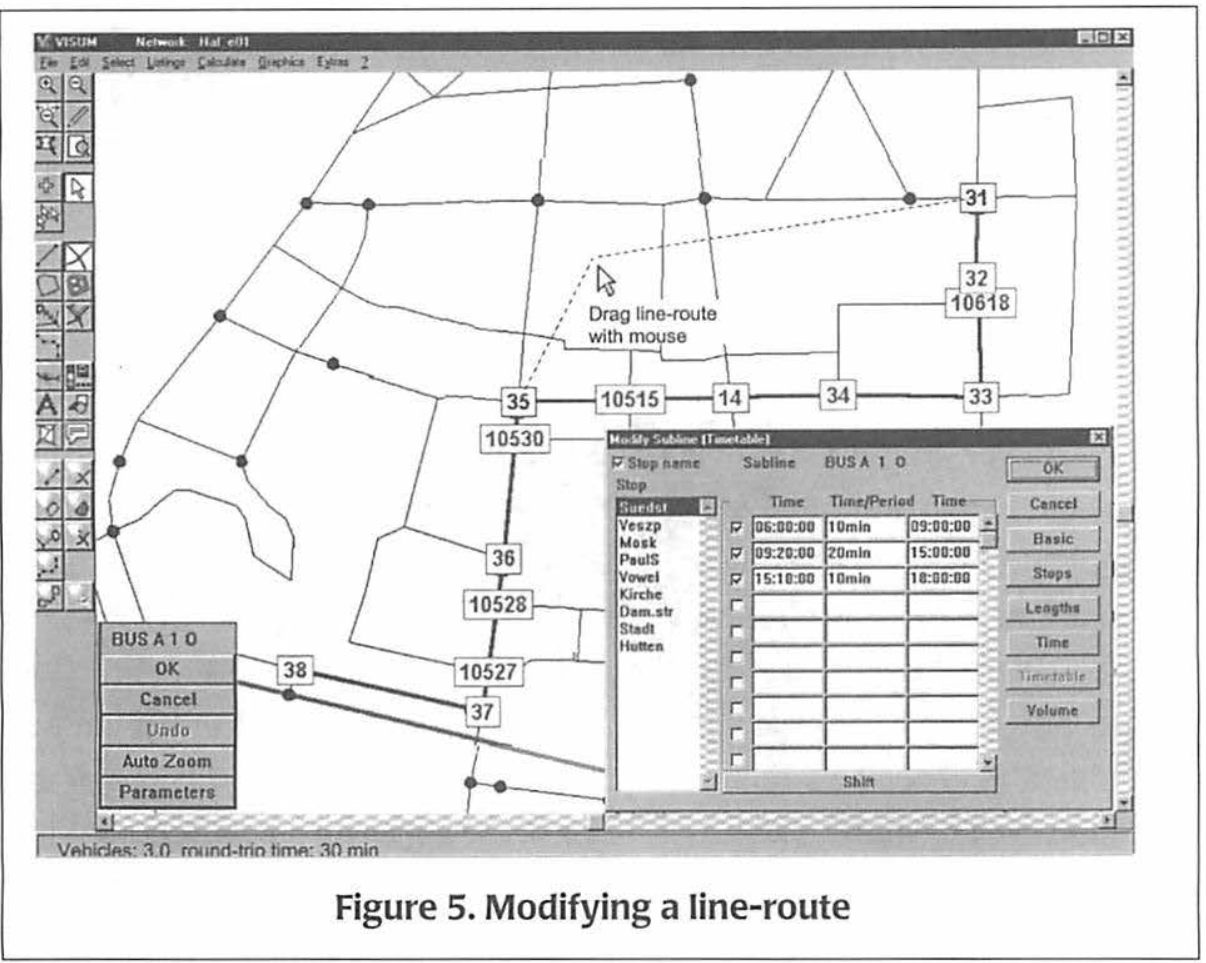

Source: Screen shot from VISUM 6.

- line length and running time,

- round-trip time and number of required vehicles, and

- O-D traffic that starts or ends within walking distance from the line's stops.

Advanced methods generate possible line-routes and optimize timetables with a fixed headway.

Line-Route Generation. This method (Sahling 1981) incorporates an objective function to minimize the number of transfers. Using a set of predefined terminals, it generates and evaluates a set of possible line-routes. The algorithm is based on an O-D matrix and the link network that may be used by lines. It considers existing lines and, therefore, allows the planner to focus on one transport system (e.g., bus) while other transport systems (e.g., train) are considered as fixed inputs. 
Timetable Optimization. This method (Guenther 1985, Maziejewski 1992) minimizes the transfer waiting time of passengers in a line network with a fixed headway. Based on the results of a public transport assignment, a genetic algorithm develops and evaluates "populations" of possible solutions by varying the departure time.

\section{User Model}

The objective of the user model is to determine the impacts of a transport supply system on travelers. Important indicators for evaluating the transport supply are journey time and travel expenses between two zones. To evaluate a public transport supply, additional indicators (e.g., number of transfers, transfer wait time, and service frequency) must be considered (Friedrich 1994).

To determine these service indicators, the passenger journeys are modeled. A private transport user chooses a convenient route for his or her journey. In addition to choosing a route, the public transport user also selects a departure time from the timetable; that is, he or she searches for a connection. While a route describes only the spatial course of a trip within a network, a connection additionally encompasses temporal constraints such as departure and arrival times at the origin stop, transfer stops, and the destination stop.

Methods to model the travel behavior are based on search algorithms that determine routes or connections between an O-D. So-called shortest path algorithms are used as search algorithms to determine the "best" route, that is, the one with the lowest impedance. Impedance can consist of times, distances, comfort restraints, and costs. Depending on the search algorithm used, the shortest path represents a route or connection. Based on the service indicators of each route/connection, the assignment then distributes the trips of an O-D pair onto the found routes or connections. As the characteristics of urban public transport and regional or interregional public transport differ, VISUM provides two special assignment procedures (Figure 6).

\section{Assignment Based on Lines}

The assignment procedure based on lines (assignment based on average headway) models each line through a sequence of stops, through the running times between the stops, and through the headway of the line. Lines with no 


\begin{tabular}{|c|c|}
\hline Assignment Based on Lines & Assignment Based on Timetables \\
\hline 1. Route Search & 1. Connection Search \\
\hline $\begin{array}{l}\text { Search for best route: } \\
\text { Impedance = } \\
\quad \text { access time + egress time + in-vehicle time } \\
\quad+\text { transfer penalty } P \times \text { no. of transfers } \\
\quad+\text { mean transfer time ( }=\text { Fac } \times \text { mean headway) } \\
\text { Repeat search with different penalties } P \text { and } \\
\text { weightings of Fac to determine several routes }\end{array}$ & $\begin{array}{l}\text { Search for best connection: } \\
\text { Impedance = } \\
\text { access time + egress time + in-vehicle time } \\
\quad+\text { transfer penalty } P \times \text { no. of transfers } \\
+ \text { actual transfer time } \\
\text { Repeat search for all possible departure times at } \\
\text { origin stop }\end{array}$ \\
\hline 2. Route Choice & 2. Connection Choice \\
\hline $\begin{array}{l}\text { Delete unattractive routes, where: } \\
\text { journey time }>\text { min. journey time } \times \text { factor } \\
\text { transfers }>\text { min. transfers }+ \text { factor }\end{array}$ & $\begin{array}{l}\text { Delete unattractive routes, where: } \\
\text { journey time }>\text { min. journey time } \times \text { factor } \\
\text { transfers }>\text { min. transfers + factor }\end{array}$ \\
\hline 3. Route Split & 3. Connection Split \\
\hline $\begin{array}{l}\text { For each route calculate: } \\
\text { - perceived journey time PJT, which consists of } \\
\text { weighted components of journey time } \\
\text { - fare }\end{array}$ & $\begin{array}{l}\text { For each connection calculate: } \\
\text { - perceived journey time PJT, which consists of } \\
\text { weighted components of journey time } \\
\text { - fare } \\
\text { - temporal utility } U \text {, which results from } \\
\text { comparing the desired departure time of } \\
\text { passengers with the actual departure times of } \\
\text { the connection } \\
\text { - impedance Imp = } f(P F T, \text { Fare, U) }\end{array}$ \\
\hline $\begin{array}{l}\text { Distribute trips with Kirchhoff Law: } \\
\qquad P_{i}=\frac{I m p_{i}^{-\alpha}}{\sum_{j=1}^{n} I m p_{j}^{-\alpha}}\end{array}$ & $\begin{array}{l}\text { proportion of trips using route/connection } i \\
\text { number of routes/connections } \\
\text { impedance of route/connection } i \\
\text { impedance sensitivity factor }\end{array}$ \\
\hline \multicolumn{2}{|c|}{$\begin{array}{c}\text { Figure 6. Characteristics of assignment based on lines } \\
\text { and assignment based on timetables }\end{array}$} \\
\hline
\end{tabular}

fixed-rhythm headway are described by their mean headway. This procedure does not explicitly calculate a transfer time but assumes that the transfer time depends on the headway. This means the coordination of the timetable is not considered. Usually the wait times at the boarding or transfer stops are equal to half of the line's headway. Assignment based on lines guarantees good assignment results for urban areas with a dense network and short headways.

\section{Assignment Based on Timetable}

The assignment procedure based on timetable (real-time assignment) con- 
siders the timetable of each transit line with its exact departure and arrival times (Friedrich 1994). A shortest-path algorithm based on these data calculates the "best" connection between two traffic zones for a particular departure time. For different times of departure, different "best" connections may be calculated that can differ by the used transit lines and/or transfer stops. To determine all "best" connections, the shortest-path algorithm is performed several times for each possible departure time within the assignment time interval. Passengers select from this set of possible connections. Their choice is influenced by the service indicators of each connection and by the utility of the departure time. The individual components of disutility (e.g., access time, transfer time, in-vehicle time, fare) are weighted with user-definable perceived unit costs. Assignment based on timetable is the appropriate method for rural areas or rail networks, where headways are long and the coordination of the timetable is important for the service quality. The exact calculation of connections, however, requires more computing time than the assignment based on lines.

\section{Assignment Results}

The assignment produces three types of results (Figure 7): traffic volumes on links, lines, and stops; service indictors for all O-D pairs; and routes. VISUM's unique feature of storing all routes during assignment allows extensive postassignment analysis of traffic flows. It can also be used to calculate revenues from passenger fares without performing a new assignment. Thus, it is possible to easily evaluate the impacts of new fare systems or higher fares concerning the revenue and cost coverage of lines.

\section{Operator Model}

To estimate the impacts on transit operators, the operator model is applied to determine indicators that express the operational and financial requirements for providing a transit service. The operator model supports line-costing calculations-a most useful tool for those responsible for strategic, financial, and operation planning. Line costing states the profit or loss on individual transit lines regarding costs and revenues.

In order to evaluate the performance of a transit line, it is necessary to deter- 


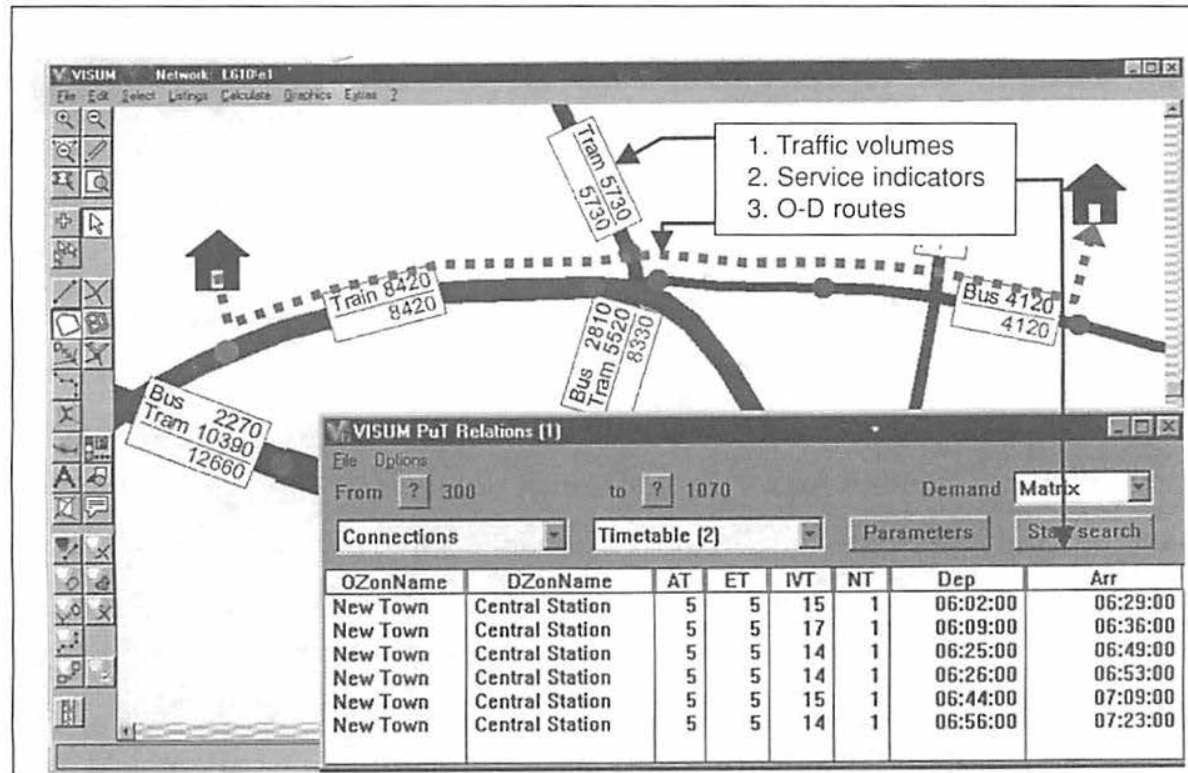

Figure 7. Assignment results: traffic volumes, service indicators, and routes

Source: Screen shot from VISUM 6.

mine indicators on a line level. This is easy for indicators such as vehicle kilometer, which can be directly calculated from the line length and the timetable. Indicators such as operating cost from vehicle depreciation or revenues from passenger fares, however, are more complicated, since a vehicle may be employed for several lines and a passenger may use more than one line for his or her journey. Operation indicators can be divided into the following categories:

- system performance indicators,

- vehicle requirement indicators,

- transport performance indicators,

- cost indicators, and

- revenue indicators.

\section{System Performance Indicators}

System performance indicators express operation requirements in kilometers or time units. They are calculated automatically after every modifica- 
tion of line data and do not require demand data. Examples of performance indicators are:

- Vehicle kilometer: Vehicle trip length $x$ number of vehicle trips.

- Service time: Time for passengers transport = line running time $x$ number of vehicle trips.

- Seat kilometer: Vehicle kilometer $x$ seats of vehicle.

\section{Vehicle Requirement Indicators}

VISUM provides an algorithm with which planners can estimate the number of required vehicles for a specified transit supply. The main goal of this calculation is to assign the total number of vehicle trips of an operational day to vehicles so that a minimum number of vehicles is required. The basis for this calculation is the timetable. It consists of individual vehicle trips that are described by subline, direction, and departure time from the first stop of the line. Vehicle rotation results from the concatenation of individual vehicle trips to trip chains that can each be performed by one vehicle. In the simplest case, a vehicle trip is concatenated at its last stop with a subsequent vehicle trip that starts at the same stop. If such a concatenation is not possible or useful, the vehicle can be redeployed to a different stop.

\section{Transport Performance Indicators}

Combining supply data with travel-demand data quantifies the transport performance described by indictors like number of boarding passengers, saturation, and passenger kilometer. These indicators are calculated automatically during assignment.

\section{Cost Indicators}

The costs of a line consist of these cost segments:

- Hourly costs: Time-dependent costs for personnel.

- Kilometer costs: Kilometer-dependent costs for fuel, repair, etc.

- Vehicle costs: Fixed costs for each required vehicle (depreciation, insurance).

- Network infrastructure costs: Costs from depreciation of new links or running costs for maintaining the network.

- Operator costs: Share of costs for overhead costs. 
Costs for vehicles, network infrastructure, or overhead need to be distributed to individual lines or vehicle trips. This requires a distribution key considering vehicle kilometer, seat kilometer, and service time.

\section{Revenue Indicators}

To estimate revenue from ticket fares, a revenue value per transported passenger is calculated considering the fare system (distance-based fare, zonebased fare). This revenue value is then distributed over the lines used by the passenger for one passenger trip. Revenue can be distributed onto the sections of a trip using the length of each trip section or the number of trip sections. Figure 8 shows an example with three different approaches to distribute a revenue of 3.00 monetary units onto two lines. Cost coverage of a line is calculated by comparing revenues and costs.

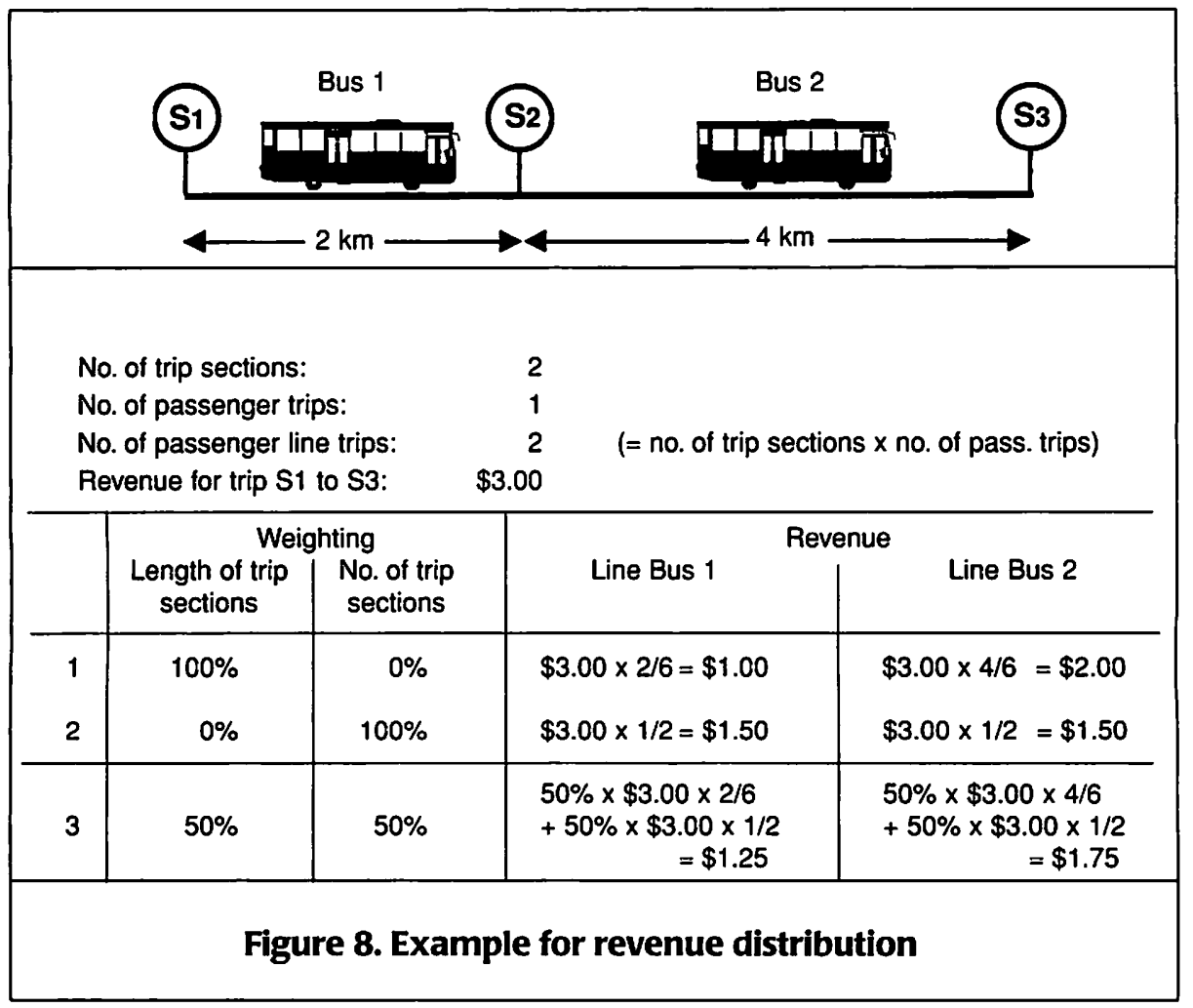




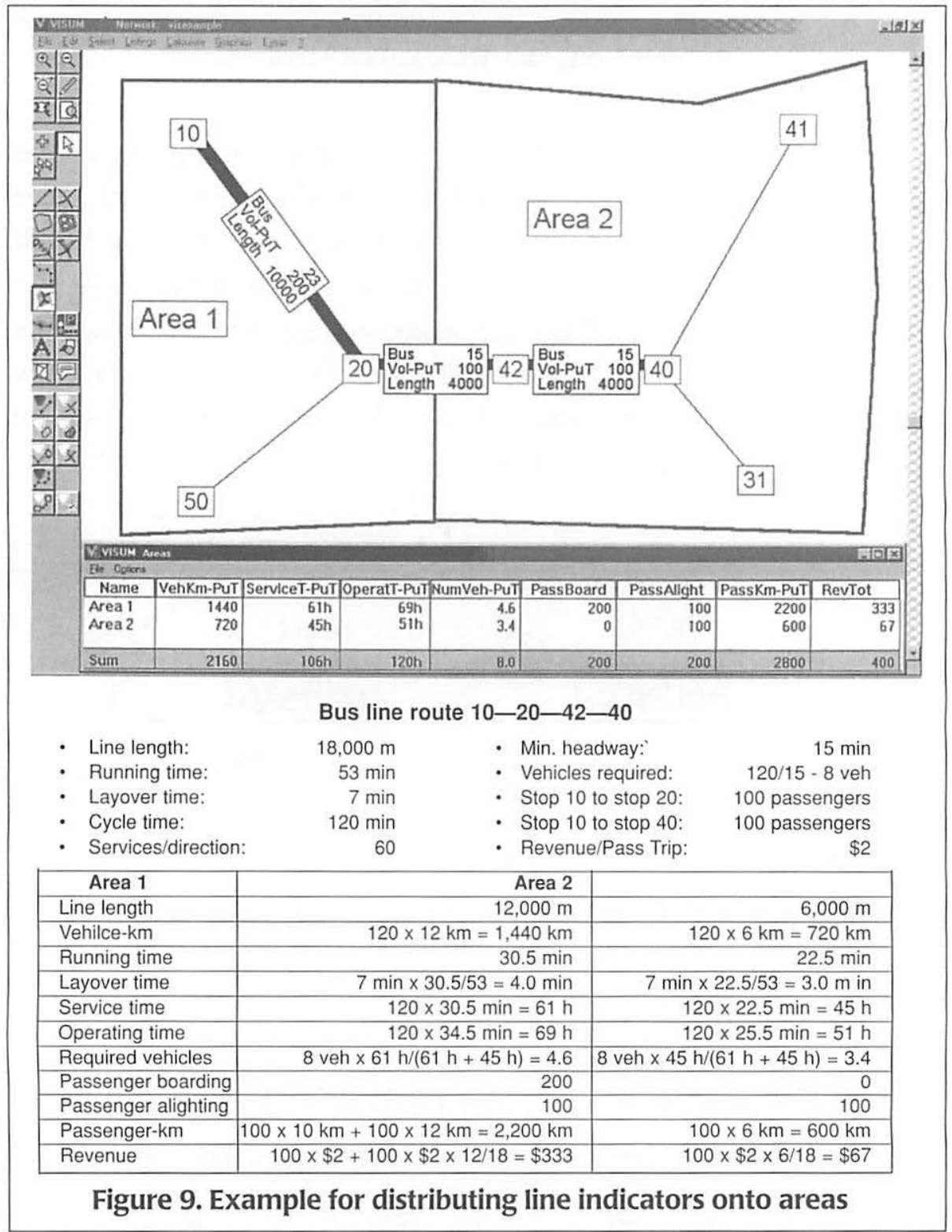

Source: Screen shot from VISUM 6. 


\section{Selected Analysis}

All performance indicators as well as cost and revenue indicators may be aggregated and displayed on the level of individual lines, supply systems, or areas (e.g., districts). In order to determine indicators for an area it is necessary to define a specific calculation routine for each indictor (Figure 9). Vehicle kilometers of a line can be distributed to areas proportional with the line length inside the area. This is not possible for the revenues of a line because a line might earn different revenues in each area. Therefore, the procedure for distributing revenues to areas must process each single passenger route with its individual revenues.

\section{Conclusions}

Demands for an efficient public transport network that offers alternatives to private transport and requires minimum public subsidies call for a planning process in which the impacts on passengers and operators are considered simultaneously. This requires a planning system that integrates:

- a disaggregated version of the four-step procedure (CTM),

- detailed transit data and specific methods to analyze the impacts of transit supply systems, and

- GIS capabilities for editing networks and analyzing spatial impacts.

VISUM combines these requirements, thus stimulating the planner within the planning process to experiment with alternative solutions. Various performance indicators are calculated concurrently with the modification of network data, so that the impacts of measures can be assessed easily. Since modifications of the transit supply directly influence operating costs and revenues, it is recommended that a line-costing calculation be included. Combining assignment results with a fare model allows measures like new fare systems and higher fares to be evaluated.

VISUM currently has almost 400 installations, mainly in Europe. VISUM is used for transit planning by cities (e.g., Berlin, Vienna), transit agencies (e.g., Dresden, VSN Group), and railway companies (e.g., DB AG, SNCF). Compared to other CTMs and GIS, VISUM supports a broad and detailed tran- 
sit function. (For a comparison of Emme/2 and VISUM, see SAMPLAN 1999.) Embedded in a multimodal model, its transit functions serve as powerful tools for planners involved in transit planning as well as integrated planning.

\section{References}

Fellendorf, M., T. Haupt, U. Heidel, and W. Scher. 1997. PTVVISION: Activity-based demand forecasting in daily practice. In Activity-based approaches to travel analysis. Oxford: Elsevier, 55-72.

Friedrich, M. 1994. Computer assisted design of public transport systems in rural areas. Schriftenreihe des Lehrsstuhls für Verkehrs-und Stadtplanung 5.

Friedrich, M. 1998. A multimodal transport model for integrated planning. Book of abstracts of the 8th World Conference on Transport Research.

Friedrich, M. 1999. Modeling public transport-A European approach, on Preprint CD-ROM of the 78th Annual Meeting. Transport Research Board.

Guenther, R. 1985. Planning and operational measures to improve connections in urban bus network. Schriftenreihe des Instituts für Verkehrsplanung und Verkehrswegebau 15.

Maziejewski, S. 1992. The vehicle routing and scheduling problem with time window constraints using genetic algorithms. Diploma thesis, Institut für Logistik, Komplexität, und Deduktionssysteme, Technical University of Karlsruhe.

Meyer, K. J. 1997. Regionalization and competition in Europe. Der nahverkehr 5/97. PTV Planung Transport Verkehr AG. 1999. www.ptv.de.

Sahling, B. M. 1981. Planning public transport lines. Institutsnotiz des Instituts für Verkehrswesen (29).

SAMPLAN. 1999. Choice of network analysis system for the new Swedish national model system SAMPERS. Swedish Institute for Transport and Communications Analysis.

UITP Union des Transports Public. 1996. Étude sur la normalisation des modèles de donnés en transport public. International Union of Public Transport.

\section{About the Authors}

MARKUS FrIEDRICH (markus.friedrich@ptv.de) directs the Transport Planning Systems Unit at PTV in Karlsruhe, Germany. He received his doctorate degree in transportation engineering from Munich University in 1994. 
Thomas Haupt (thomas.haupt@ptv.de) is director of the Business Transportation Modeling Unit at PTV.

KLAUS NOEKEL (klaus.noekel@ptv.de) is responsible for research and development at PTV. He received his doctorate degree in computer science from the University of Kaiserslautern, Germany, in 1990. 